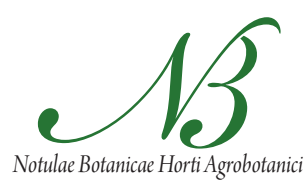

Cluj-Napoca

\title{
Studies on Genetic Variability and Correlation among the Different Traits in Solanum lycopersicum L.
}

\author{
Iraj BERNOUSI ${ }^{1}$, Aliyeh EMAMI ${ }^{2}$, Mehdi TAJBAKHSH ${ }^{1}$, Reza \\ DARVISHZADEH ${ }^{1,3,}$, Mashhid HENAREH $^{2}$ \\ ${ }^{1}$ University of Urmia, Faculty of Agriculture, Department of Agronomy and Plant Breeding, \\ Urmia,Iran;i.bernosi@urmia.ac.ir,m.tajbakhsh@urmia.ac.ir \\ ${ }^{2}$ West Azerbaijan Agricultural and Natural Resources Research Center, Urmia, Iran \\ ${ }^{3}$ University of Urmia, Institute of Biotechnology,Urmia, Iran; r.darvishzadeh@mail.urmia.ac.ir ('corresponding author)
}

\begin{abstract}
In present study the genetic diversity in some tomato genotypes were investigated in two years at North-west of Iran where due to its geographical situation is one of the most favourable regions for tomato cultivation. Twenty five tomato genotypes were evaluated for yield and important morphological traits during 2006-2007. Experiment was conducted in a $5 \times 5$ lattice square design with 3 replications in each one of years. Data on morphological traits were collected from central row on individual plants basis on mean values of five plants of each genotype selected at random. Analysis of variance on the studied traits revealed significant differences among genotypes for all the characters except for fruit yield, total soluble solids, titratable acidity and number of tillers. Genotype $\times$ year interaction was only significant for some of the characters including number of fruit per plant, titratable acidity and for fruit yield. Mean data revealed high range for most of studied traits. Maximum and minimum variability were observed for number of fruit per plant and $\mathrm{pH}$ respectively. Hierarchical cluster analysis allowed the assessment of similarity and clarified some of the relationships among tomato genotypes. UPGMA produced a dendrogram with four clusters. The first cluster included $64 \%$ of studied tomato genotypes. Results from the PCA indicated that more than $91 \%$ of the variability observed can be explained by the first seven components. Based on PCA, $\mathrm{PC} 1$ can be considered as representatives of fruit size, while properties that show high correlation with PC2 are the ones related to plant characteristics.
\end{abstract}

Keywords: cluster analysis, combined analysis, genetic diversity, principal component analysis, tomato

\section{Introduction}

Tomato (Solanum lycopersicum L., formerly Lycopersicon esculentum Miller) is an economically important crop worldwide. It has a diploid genome with 12 chromosome pairs and a genome size of $950 \mathrm{Mb}$ (Michaelson et al., 1991) encoding approximately 35,000 genes that are largely sequestered in contiguous euchromatic regions (Van der Hoeven et al., 2002). Tomato has its primary center of diversity in a narrow belt along the Andean region of Ecuador and Peru (Carelli et al., 2006). A commonly accepted hypothesis for the domestication of cultivated tomato is that: (1) the $S$. lycopersicum subsp. cerasiforme spread as a weed from the Andean region to Mexico, where it was domesticated; (2) the domesticate tomato was taken to $\mathrm{Eu}$ rope in the sixteenth century; (3) it was then disseminated to many areas of the world (Rick, 1976). During its evolution and domestication $S$. lycopersicum has undergone various genetic 'bottlenecks' imposed by self-pollination, founder effects, artificial and natural selection, and extreme inbreeding of limited genotypes, particularly in $\mathrm{Eu}-$ rope and North America (Rick, 1991). The initial narrow genetic basis of the tomato was further restricted by the development of vintage and modern cultivars, when much of the diversity within the cultivated $S$. lycopersicum was lost (Rick, 1976; Miller and Tanksley, 1990; Williams and St. Clair, 1993).

One of the pre-requirements for successful breeding strategies is the complete understanding of the genetic diversity of the crop plant. Morphological characteristics are the strongest determinants of the agronomic value and taxonomic classification of plants. Compared with other means, morphological evaluations are direct, inexpensive and easy. However, errors can arise; furthermore, morphological estimations are more dependent on environment and are more subjective than other measurements. The reliability of measurements can be improved by repeating experiments in several environments. Systematic study and evaluation of tomato germplasm is of great importance for current and future agronomic and genetic improvement of the crop. Furthermore, if an improvement programme is to be carried out, evaluation of germplasm is imperative, in order to understand the genetic background and the breeding value of the available germplasm (Agong et al., 
2000). The genetic variability is the raw material of vegetable breeding industry on which selection acts to evolve superior genotypes. The higher amount of variation present for a character in the breeding materials, greater is the scope for its improvement through selection. In tomato, yield is the cumulative effect of many component characters individually contributing towards yield. The knowledge of association of fruit yield with its component traits helps in achieving success in a breeding programme. Singh et al. (2002) observed high genetic variation for plant height, number of days to fruit set, number of fruit clusters per plant, number of fruits per plant, fruit weight per plant and fruit yield per plant. The amount of association between characters with yield can be ascertained by correlation studies. This would aid in formulating an efficient breeding program for improving the yield potential via its components.

Tomato is one of the most popular and important vegetables in Iran. It is cultivated in all parts of the country. This study presents the first report on the investigation of genetic differences for different traits in some tomato genotypes investigated in two years at North-west of Iran where due to its geographical situation is one of the most favourable regions for tomato cultivation.

\section{Material and methods}

\section{Plant materials and experimental design}

Twenty five tomato genotypes available in the Plant Genetic Resources Center at West Azerbaijan Agricultural and Natural Resources Research Institute, Iran were evaluated for yield and important morphological traits during 2006-2007. Genotypes include: 'To4' (1), 'Chase' (2), 'Carmina' (3), 'King Stone' (4), 'Nina' 115 (5), 'C H Falat' (6), 'Super Srin B' (7), 'Primo Early' (8), 'Primo Falat' (9), 'Pto Early' C H (10), 'Early Orbana V F' (11), 'Pri Max' (12), 'Cal J' (13), 'Shaf Falat' (14), 'Salab Jino' (15), 'Cal J N' (16), 'Early Orbana 111' (17), 'Shaf' (18), 'Early Orbana Y' (19), 'Super Ston' (20), 'Y Falat' (21), 'Bss-282' (22), 'Tima' (23), 'To2' (24) and 'Falat 111' (25). The seed beds were prepared in March. After sowing the seeds, a fine layer of well fermented and sieved sheep manure was spread on top of beds. Just prior to transplanting, the field were more thoroughly ploughed and disked to break up the soil and smooth and level the field. Tomato seedlings were transplanted to field when plant averaged about $12 \mathrm{~cm}$ in height. The experiment was conducted in a $5 \times 5$ lattice square design with 3 replications in each one of years. Each plot was comprised of 3 lines of $5 \mathrm{~m}$. Row to row and plant to plant spacing was kept 1.20 and $0.40 \mathrm{~m}$, respectively. About three weeks after transplanting, once the plants were well established, using a shorthandled hoe, the soil around each plant was loosened and any weeds removed. Fields were fertilized with $\mathrm{N}-\mathrm{P}-\mathrm{K}$ (basal application and side-dressings) based on standard crop production technology as needed for tomato crop in this region. Field irrigation was carrying out after $80 \%$ depletion of soil available water. Insects were controlled with pesticides. Harvests were performed when fruits reached the completely ripe stage, with $100 \%$ of their surface presenting an intense red coloration. The addition of the all harvests resulted in the marketable production. The evaluation of fruit quality characteristics was carried through in composed sample, originated from at least four plants in each replicates, with fruits harvested in the third cluster. In this experiment, only the marketable standard fruits were considered as marketable yield, disregarding fruits with blemishes, and attacked by fungal or bacterial diseases. The following fruit characteristics were evaluated: titratable acidity (TA, \% of citric acid), $\mathrm{pH}$ and total soluble solids (TSS, Brix). Data on morphological traits were collected from central row on individual plant basis on mean values of five plants of each genotype selected at random for the following characters: number of fruit per plant (NF), mean of fruit weight per plant (FW), fruit length (FL), fruit diameter (FD), pericarp thickness (PT), number of carpel (NC), leaflet width (LletW), leaflet length (LletL), principal leaf length (LL), plant height (PHeight), number of node on stem (NNS), number of tiller (NT), number of floret (NFlo), number of days to flowering (DFlow) and number of days to fruit production (DFP).

\section{Statistical analysis}

Normality of the data was assessed according to the Shapiro and Wilk test (PROC UNIVARIATE of SAS software; (SAS Institute, Cary, NC). The significant of variability among 25 tomato genotypes was tested through combined analyses of $5 \times 5$ lattice square designs. The purpose of the combined analysis was to identify the effect of genotype and environment as well as their interactions on the studied traits. Correlations between fruit yield per plant and different characters were determined in the SAS software (SAS Institute, Cary, NC). Clustering of genotypes into similarity groups was performed using the method of UPGMA (un-weighted pair-grouped method with arithmetic average). In order to identify the patterns of morphological variation, principal component analysis (PCA) was conducted. This part of data processing was performed using statistic program 'Minitab' version 14.

\section{Results and discussion}

Analysis of variance on the studied traits presented in the Tab. 1 revealed significant differences among genotypes for all the characters except for fruit yield, total soluble solids, titratable acidity and number of tillers. The effect of year for various characters (leaflet width, principal leaf length, leaflet length, total soluble solids, titratable acidity, plant height, number of node on stem and number of tillers) indicated the influence of environmental changes over the years that were expected under field conditions in a crop like tomato. These differences 


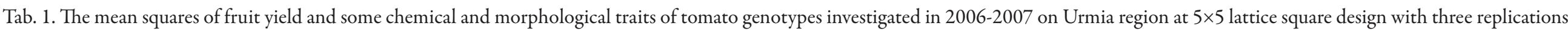

\begin{tabular}{|c|c|c|c|c|c|c|c|c|c|c|c|c|c|c|c|c|c|c|c|c|}
\hline \multirow{2}{*}{ S.O.V } & \multirow{2}{*}{$\mathrm{df}$} & \multicolumn{19}{|c|}{ MS } \\
\hline & & Yield & NF & FW & FL & FD & PT & $\mathrm{NC}$ & LletW & $\mathrm{LL}$ & LletL & TSS & $\mathrm{pH}$ & TA & PHeight & NNS & NT & NFlo & DFlow & DFP \\
\hline Year & 1 & $150.89^{\mathrm{ns}}$ & $0.39^{\mathrm{ns}}$ & $685.51^{\mathrm{ns}}$ & $0.05^{\mathrm{ns}}$ & $0.47^{\mathrm{ns}}$ & $0.0002^{\mathrm{ns}}$ & $0.92^{\mathrm{ns}}$ & $33.01^{*}$ & $592.38^{\prime \prime}$ & $37.01^{\circ}$ & $14.80^{\prime \prime}$ & $2.16^{\mathrm{ns}}$ & $4.01^{*}$ & 14625 & $2441.51^{\prime \prime}$ & $0.05^{\prime \prime}$ & $10.44^{\mathrm{ns}}$ & $860.77^{\prime \prime}$ & $39.87^{\mathrm{ns}}$ \\
\hline Rep(Year) & 4 & $8.29^{\mathrm{ns}}$ & $67.48^{\text {ns }}$ & $32.17^{\text {ns }}$ & $0.13^{\mathrm{ns}}$ & $0.51^{\mathrm{ns}}$ & $0.01^{\mathrm{ns}}$ & $0.63^{*}$ & 0.59 & $19.89^{\text {ns }}$ & $2.53^{\mathrm{ns}}$ & $0.11^{\mathrm{ns}}$ & $0.005^{\mathrm{ns}}$ & $0.005^{\mathrm{ns}}$ & $388.89^{\text {ns }}$ & $5.12^{\mathrm{ns}}$ & $0.33^{\text {ns }}$ & $0.50^{\mathrm{ns}}$ & $24.86^{\circ}$ & $30.85^{\mathrm{ns}}$ \\
\hline Genotype & 24 & $58.82^{\text {ns }}$ & $11007.00^{*}$ & $1330.72^{*}$ & $2.94^{\prime \prime}$ & $1.14^{\prime \prime}$ & $0.03^{\prime \prime}$ & $2.13^{*}$ & $1.40^{*}$ & $18.54^{\prime \prime}$ & $3.01^{*}$ & $0.30^{\mathrm{ns}}$ & 0.04 & $0.03^{\mathrm{ns}}$ & $1553.15^{\prime \prime}$ & $34.32^{\prime \prime}$ & $0.70^{\text {ns }}$ & $3.65^{\prime \prime}$ & $10.06^{\mathrm{ns}}$ & $70.68^{\prime \prime}$ \\
\hline Year×Genotype & 24 & $100.43^{\prime \prime}$ & $412.02^{*}$ & $132.61^{\mathrm{ns}}$ & $0.17^{\mathrm{ns}}$ & $0.10^{\mathrm{ns}}$ & $0.006^{\mathrm{ns}}$ & $0.39^{\mathrm{ns}}$ & $0.38^{\mathrm{ns}}$ & $6.42^{\mathrm{ns}}$ & $0.66^{\mathrm{ns}}$ & $0.16^{\mathrm{ns}}$ & $0.01^{\mathrm{ns}}$ & $0.02^{\circ}$ & $53.70^{\mathrm{ns}}$ & $2.87^{\mathrm{ns}}$ & $0.41^{\mathrm{ns}}$ & $0.34^{\mathrm{ns}}$ & $10.31^{\mathrm{ns}}$ & $16.36^{\mathrm{ns}}$ \\
\hline $\mathrm{Col} \times \operatorname{Rep}($ Year $)$ & 24 & $26.00^{\mathrm{ns}}$ & $34.59^{\mathrm{ns}}$ & $50.35^{\mathrm{ns}}$ & $0.21^{\mathrm{ns}}$ & $0.13^{\mathrm{ns}}$ & $0.006^{\mathrm{ns}}$ & $0.22^{\mathrm{ns}}$ & $0.31^{\mathrm{ns}}$ & $4.51^{\mathrm{ns}}$ & $0.78^{\mathrm{ns}}$ & $0.11^{\mathrm{ns}}$ & $0.01^{\mathrm{ns}}$ & $0.008^{\mathrm{ns}}$ & $100.58^{\mathrm{ns}}$ & $3.08^{\mathrm{ns}}$ & $0.84^{\mathrm{ns}}$ & $0.17^{\mathrm{ns}}$ & $4.28^{\mathrm{ns}}$ & $8.47^{\mathrm{ns}}$ \\
\hline Row $\times \operatorname{Rep}($ Year $)$ & 24 & $33.21^{\mathrm{ns}}$ & $99.09^{\text {ns }}$ & $162.10^{\text {ns }}$ & $0.27^{\mathrm{ns}}$ & $0.36^{\mathrm{ns}}$ & $0.009^{\text {ns }}$ & $0.38^{\mathrm{ns}}$ & $0.82^{\circ}$ & $12.74 "$ & $1.40^{\mathrm{ns}}$ & $0.28^{\prime \prime}$ & $0.008^{\text {ns }}$ & $0.01^{\mathrm{ns}}$ & $95.88^{\text {ns }}$ & $4.48^{\mathrm{ns}}$ & $0.67^{\mathrm{ns}}$ & $0.72^{\mathrm{ns}}$ & $8.03^{\mathrm{ns}}$ & $10.80^{\mathrm{ns}}$ \\
\hline Error & 48 & 31.26 & 69.58 & 100.30 & 0.21 & 0.21 & 0.007 & 0.53 & 0.38 & 4.80 & 0.82 & 0.12 & 0.01 & 0.009 & 119.50 & 3.83 & 1.03 & 0.60 & 9.86 & 11.13 \\
\hline $\mathrm{CV}$ & & 9.82 & 13.93 & 11.49 & 8.02 & 9.29 & 12.65 & 18.55 & 11.41 & 7.59 & 9.02 & 7.17 & 2.56 & 16.78 & 13.69 & 14.50 & 20.26 & 20.60 & 3.72 & 2.74 \\
\hline
\end{tabular}

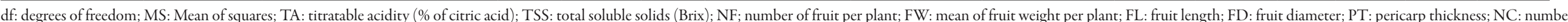

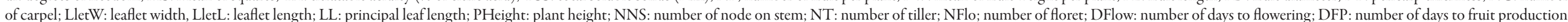

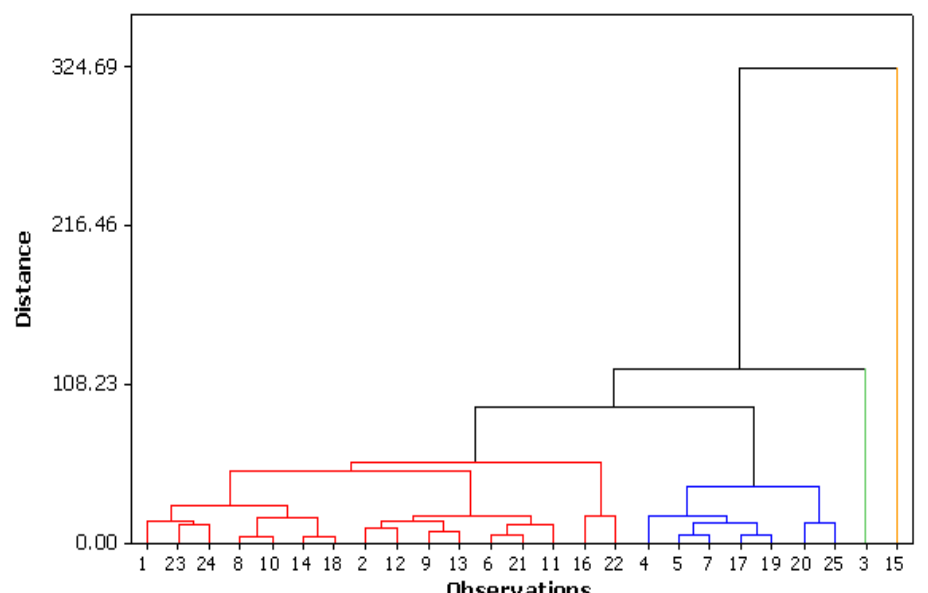

Fig. 1. Dendogram for the 25 tomato genotypes obtained from different regions, produced by Ward's clusters analysis; clusters are based on morphological traits (scale: Squared Euclidean distance). 'To4' (1), 'Chase' (2), 'Carmina' (3), 'King Stone' (4), 'Nina' 115 (5), 'C H Falat' (6), 'Super Srin B' (7), 'Primo Early' (8), 'Primo Falat' (9), 'Pto Early' C H (10), 'Early Orbana V F' (11), 'Pri Max' (12), 'Ca J' (13), 'Shaf Falat' (14), 'Salab Jino' (15), 'Cal J N' (16), 'Early Orbana 111' (17), 'Shaf' (18), 'Early Orbana Y' (19), 'Super Ston' (20), 'Y Falat' (21), 'Bss-282' (22), 'Tima' (23), 'To2' (24) and 'Falat $111^{\prime}(25)$

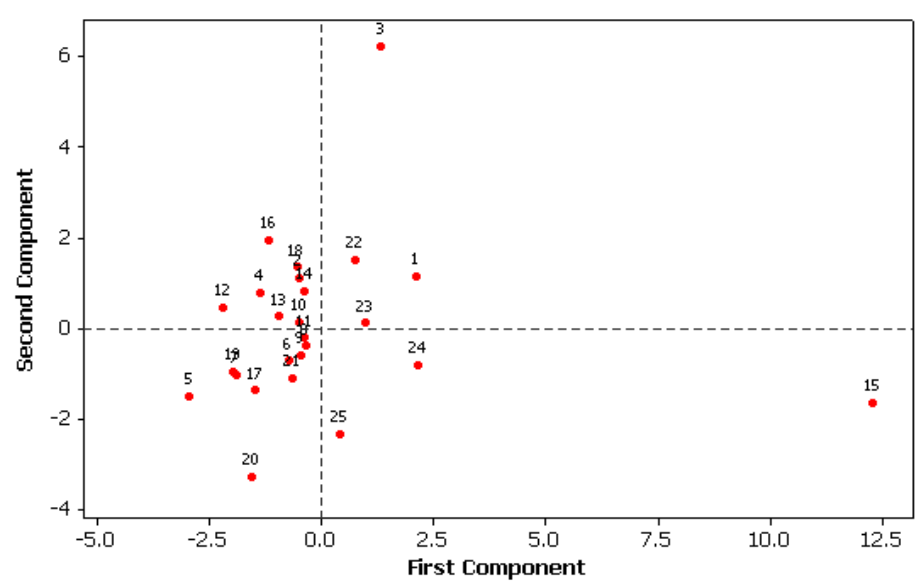

Fig. 2. Relationships among 25 tomato genotypes shown by a $2 \mathrm{D}$ scatter for first two principal components based on morphological traits. 'To4' (1), 'Chase' (2), 'Carmina' (3), 'King Stone' (4), 'Nina' 115 (5), 'C H Falat' (6), 'Super Srin B' (7), 'Primo Early' (8), 'Primo Falat' (9), 'Pto Early' C H (10), 'Early Orbana V F' (11), 'Pri Max' (12), 'Cal J' (13), 'Shaf Falat' (14), 'Salab Jino' (15), 'Cal J N' (16), 'Early Orbana 111' (17), 'Shaf' (18), 'Early Orbana Y' (19), 'Super Ston' (20), 'Y Falat' (21), 'Bss-282' (22), 'Tima' (23), 'To2' (24) and 'Falat 111 ' (25) 
Tab. 2. Average fruit yield and some chemical and morphological traits of 25 tomato genotypes investigated in $2006-2007$ on Urmia region at $5 \times 5$ lattice square design with three replications

\begin{tabular}{|c|c|c|c|c|c|c|c|c|c|c|c|c|c|c|c|c|c|c|c|}
\hline 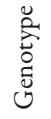 & Yield & NF & FW & FL & FD & PT & $\mathrm{NC}$ & LletW & LL & LletL & TSS & $\mathrm{pH}$ & TA & PHeight & NNS & NT & NFlo & DFlow & DFP \\
\hline 1 & $59.63 \pm 2.78$ & $71.28 \pm 4.15$ & $71.33 \pm 4.98$ & $4.93 \pm 0.22$ & $4.57 \pm 0.23$ & $62 \pm 0.04$ & $4.54 \pm 0.36$ & $4.87 \pm 0.31$ & $28.31 \pm 1.09$ & $9.57 \pm 0.45$ & $5.13 \pm 0.17$ & $4.16 \pm 0.05$ & $0.60 \pm 0.05$ & $82.81 \pm 5.43$ & $14.47 \pm 0.97$ & $5.41 \pm 0.51$ & $3.77 \pm 0.38$ & $82.38 \pm 1.56$ & $115.52 \pm 1.66$ \\
\hline 2 & $51.59 \pm 2.78$ & $48.14 \pm 4.15$ & $81.32 \pm 4.99$ & $5.89 \pm 0.23$ & $45 \pm 0.23$ & $72 \pm 0.04$ & $3.86 \pm 0.36$ & $4.61 \pm 0.31$ & $4.71 \pm 1.09$ & $9.06 \pm 0.45$ & $4.83 \pm 0.17$ & $4.10 \pm 0.05$ & $0.58 \pm 0.05$ & $68.53 \pm 5.44$ & $12.56 \pm 0.98$ & $5.18 \pm 0.51$ & $2.96 \pm 0.38$ & $85.04 \pm 1.56$ & $121.76 \pm 1.66$ \\
\hline 3 & $58.42 \pm 2.78$ & $53.38 \pm 4.15$ & $88.53 \pm 4.98$ & $4.52 \pm 0.22$ & $5.56 \pm 0.23$ & $0.71 \pm 0.04$ & $3.96 \pm 0.36$ & $4.29 \pm 0.31$ & $31.33 \pm 1.09$ & $8.25 \pm 0.45$ & $5.17 \pm 0.17$ & $4.19 \pm 0.05$ & $0.57 \pm 0.05$ & $168.03 \pm 5.43$ & $26.43 \pm 0.97$ & $5.37 \pm 0.51$ & $4.36 \pm 0.38$ & $84.73 \pm 1.56$ & $117.42 \pm 1.66$ \\
\hline 4 & $57.25 \pm 2.78$ & $45.48 \pm 4.15$ & $112.48 \pm 4.98$ & $5.49 \pm 0.22$ & $5.31 \pm 0.23$ & $0.62 \pm 0.04$ & $4.95 \pm 0.36$ & $5.33 \pm 0.31$ & $30.59 \pm 1.09$ & $8.95 \pm 0.45$ & $4.76 \pm 0.17$ & $4.15 \pm 0.05$ & $0.50 \pm 0.05$ & $89.80 \pm 5.43$ & $15.64 \pm 0.97$ & $4.68 \pm 0.51$ & $3.53 \pm 0.38$ & $85.98 \pm 1.56$ & $123.12 \pm 1.66$ \\
\hline 5 & $52.94 \pm 2.78$ & $46.29 \pm 4.15$ & $99.60 \pm 4.99$ & $6.39 \pm 0.23$ & $5.18 \pm 0.23$ & $0.69 \pm 0.04$ & $4.67 \pm 0.36$ & $5.61 \pm 0.31$ & $30.78 \pm 1.09$ & $11.10 \pm 0.45$ & $4.43 \pm 0.17$ & $4.17 \pm 0.05$ & $0.47 \pm 0.05$ & $74.30 \pm 5.44$ & $12.29 \pm 0.98$ & $4.53 \pm 0.51$ & $3.93 \pm 0.38$ & $87.49 \pm 1.56$ & $127.06 \pm 1.66$ \\
\hline 6 & $52.01 \pm 2.78$ & $44.99 \pm 4.15$ & $85.92 \pm 4.99$ & $5.45 \pm 0.23$ & $5.19 \pm 0.23$ & $0.66 \pm 0.04$ & $4.22 \pm 0.36$ & $5.84 \pm 0.31$ & $27.68 \pm 1.09$ & $23 \pm 0.45$ & $4.72 \pm 0.17$ & $4.17 \pm 0.05$ & $0.60 \pm 0.05$ & $66.11 \pm 5.44$ & $11.90 \pm 0.98$ & $5.23 \pm 0.51$ & $3.78 \pm 0.38$ & $84.89 \pm 1.56$ & $123.81 \pm 1.66$ \\
\hline 7 & $55.77 \pm 2.78$ & $45.68 \pm 4.15$ & $86.13 \pm 4.98$ & $5.99 \pm 0.22$ & $5.08 \pm 0.23$ & $0.64 \pm 0.04$ & $4.64 \pm 0.36$ & $5.90 \pm 0.31$ & $30.70 \pm 1.09$ & $10.45 \pm 0.45$ & $4.47 \pm 0.17$ & $4.13 \pm 0.05$ & $0.53 \pm 0.05$ & $76.39 \pm 5.43$ & $12.82 \pm 0.97$ & $5.11 \pm 0.51$ & $3.41 \pm 0.38$ & $86.33 \pm 1.56$ & $127.97 \pm 1.66$ \\
\hline 8 & $60.11 \pm 2.59$ & $43.71 \pm 3.87$ & $97.36 \pm 4.65$ & $5.84 \pm 0.21$ & $5.22 \pm 0.21$ & $0.67 \pm 0.04$ & $4.32 \pm 0.34$ & $5.97 \pm 0.29$ & $28.67 \pm 1.02$ & $10.59 \pm 0.42$ & $4.79 \pm 0.17$ & $4.12 \pm 0.05$ & $0.56 \pm 0.04$ & $77.51 \pm 5.07$ & $14.14 \pm 0.91$ & $5.27 \pm 0.47$ & $3.69 \pm 0.36$ & $83.16 \pm 1.46$ & $120.51 \pm 1.55$ \\
\hline 9 & $53.89 \pm 2.78$ & $52.04 \pm 4.15$ & $97.27 \pm 4.99$ & $5.27 \pm 0.23$ & $4.76 \pm 0.23$ & $0.67 \pm 0.04$ & $4.44 \pm 0.36$ & $5.30 \pm 0.31$ & $27.37 \pm 1.09$ & $9.86 \pm 0.45$ & $4.89 \pm 0.17$ & $4.16 \pm 0.05$ & $0.51 \pm 0.05$ & $69.15 \pm 5.44$ & $10.76 \pm 0.98$ & $4.73 \pm 0.51$ & $3.79 \pm 0.38$ & $83.84 \pm 1.56$ & $123.16 \pm 1.66$ \\
\hline 10 & $58.49 \pm 2.78$ & $43.43 \pm 4.15$ & $90.60 \pm 4.98$ & $6.04 \pm 0.22$ & $5.32 \pm 0.23$ & $0.68 \pm 0.04$ & $4.26 \pm 0.36$ & $5.75 \pm 0.31$ & $26.64 \pm 1.09$ & $9.70 \pm 0.45$ & $4.75 \pm 0.17$ & $4.22 \pm 0.05$ & $0.56 \pm 0.05$ & $78.20 \pm 5.43$ & $12.78 \pm 0.97$ & $5.03 \pm 0.51$ & $3.56 \pm 0.38$ & $81.83 \pm 1.56$ & $121.22 \pm 1.66$ \\
\hline 11 & $50.73 \pm 2.78$ & $53.39 \pm 4.15$ & $76.72 \pm 4.99$ & $5.30 \pm 0.23$ & $5.13 \pm 0.23$ & $0.66 \pm 0.04$ & $4.10 \pm 0.36$ & $5.14 \pm 0.31$ & $31.30 \pm 1.09$ & $10.36 \pm 0.45$ & $4.63 \pm 0.17$ & $4.08 \pm 0.05$ & $0.65 \pm 0.05$ & $77.79 \pm 5.44$ & $14.35 \pm 0.98$ & $5.00 \pm 0.51$ & $2.69 \pm 0.38$ & $85.04 \pm 1.56$ & $122.46 \pm 1.66$ \\
\hline 12 & $50.33 \pm 2.78$ & $40.99 \pm 4.15$ & $86.70 \pm 4.99$ & $5.63 \pm 0.23$ & $5.35 \pm 0.23$ & $70 \pm 0.04$ & $4.44 \pm 0.36$ & $5.13 \pm 0.31$ & $27.95 \pm 1.09$ & $9.65 \pm 0.45$ & $4.70 \pm 0.17$ & $4.23 \pm 0.05$ & $0.50 \pm 0.05$ & $74.89 \pm 5.44$ & $12.20 \pm 0.98$ & $5.08 \pm 0.51$ & $2.66 \pm 0.38$ & $87.29 \pm 1.56$ & $123.56 \pm 1.66$ \\
\hline 13 & $53.41 \pm 2.78$ & $53.78 \pm 4.15$ & $83.85 \pm 4.98$ & $5.80 \pm 0.22$ & $5.09 \pm 0.23$ & $0.81 \pm 0.04$ & $3.43 \pm 0.36$ & $4.82 \pm 0.31$ & $29.14 \pm 1.09$ & $10.17 \pm 0.45$ & $4.76 \pm 0.17$ & $4.20 \pm 0.05$ & $0.53 \pm 0.05$ & $74.78 \pm 5.43$ & $11.65 \pm 0.97$ & $4.74 \pm 0.51$ & $3.76 \pm 0.38$ & $83.63 \pm 1.56$ & $122.97 \pm 1.66$ \\
\hline 14 & $63.50 \pm 2.78$ & $42.93 \pm 4.15$ & $102.75 \pm 4.98$ & $5.88 \pm 0.22$ & $4.94 \pm 0.23$ & $0.67 \pm 0.04$ & $3.78 \pm 0.36$ & $5.01 \pm 0.31$ & $26.24 \pm 1.09$ & $9.11 \pm 0.45$ & $4.68 \pm 0.17$ & $4.17 \pm 0.05$ & $0.60 \pm 0.05$ & $69.22 \pm 5.43$ & $12.85 \pm 0.97$ & $5.03 \pm 0.51$ & $3.30 \pm 0.38$ & $84.58 \pm 1.56$ & $122.97 \pm 1.66$ \\
\hline 15 & $60.12 \pm 2.78$ & $308.98 \pm 4.15$ & $14.60 \pm 4.98$ & $2.68 \pm 0.22$ & $2.82 \pm 0.23$ & $0.36 \pm 0.04$ & $2.31 \pm 0.36$ & $5.51 \pm 0.31$ & $27.17 \pm 1.09$ & $9.34 \pm 0.45$ & $5.60 \pm 0.17$ & $3.78 \pm 0.05$ & $0.85 \pm 0.05$ & $88.49 \pm 5.43$ & $14.02 \pm 0.97$ & $4.78 \pm 0.51$ & $7.92 \pm 0.38$ & $81.33 \pm 1.56$ & $107.67 \pm 1.66$ \\
\hline 16 & $60.93 \pm 2.78$ & $49.48 \pm 4.15$ & $100.45 \pm 4.98$ & $6.44 \pm 0.22$ & $4.78 \pm 0.23$ & $0.83 \pm 0.04$ & $2.30 \pm 0.36$ & $4.79 \pm 0.31$ & $28.49 \pm 1.09$ & $9.65 \pm 0.45$ & $4.90 \pm 0.17$ & $4.22 \pm 0.05$ & $0.49 \pm 0.05$ & $86.95 \pm 5.43$ & $13.88 \pm 0.97$ & $5.37 \pm 0.51$ & $4.16 \pm 0.38$ & $85.78 \pm 1.56$ & $127.47 \pm 1.66$ \\
\hline 17 & $60.90 \pm 2.78$ & $49.83 \pm 4.15$ & $102.00 \pm 4.98$ & $6.60 \pm 0.22$ & $5.21 \pm 0.23$ & $0.67 \pm 0.04$ & $4.11 \pm 0.36$ & $5.96 \pm 0.31$ & $31.45 \pm 1.09$ & $11.00 \pm 0.45$ & $4.76 \pm 0.17$ & $4.08 \pm 0.05$ & $0.48 \pm 0.05$ & $74.10 \pm 5.43$ & $12.60 \pm 0.97$ & $4.93 \pm 0.51$ & $3.66 \pm 0.38$ & $84.83 \pm 1.56$ & $124.17 \pm 1.66$ \\
\hline 18 & $58.62 \pm 3.20$ & $40.44 \pm 4.78$ & $98.49 \pm 5.74$ & $5.87 \pm 0.26$ & $5.02 \pm 0.26$ & $0.73 \pm 0.05$ & $4.59 \pm 0.42$ & $4.72 \pm 0.35$ & $24.90 \pm 1.25$ & $8.75 \pm 0.52$ & $4.61 \pm 0.20$ & $4.18 \pm 0.06$ & $0.61 \pm 0.05$ & $71.70 \pm 6.26$ & $13.67 \pm 1.12$ & $4.87 \pm 0.58$ & $3.57 \pm 0.44$ & $83.92 \pm 1.80$ & $121.13 \pm 1.91$ \\
\hline 19 & $59.90 \pm 2.78$ & $48.08 \pm 4.15$ & $89.68 \pm 4.98$ & $6.01 \pm 0.22$ & $5.24 \pm 0.23$ & $0.70 \pm 0.04$ & $4.35 \pm 0.36$ & $5.70 \pm 0.31$ & $31.70 \pm 1.09$ & $11.03 \pm 0.45$ & $4.34 \pm 0.17$ & $4.17 \pm 0.05$ & $0.45 \pm 0.05$ & $73.22 \pm 5.43$ & $12.34 \pm 0.97$ & $5.37 \pm 0.51$ & $3.67 \pm 0.38$ & $83.93 \pm 1.56$ & $124.57 \pm 1.66$ \\
\hline 20 & $54.66 \pm 2.78$ & $41.63 \pm 4.15$ & $102.98 \pm 4.98$ & $6.05 \pm 0.22$ & $5.04 \pm 0.23$ & $62 \pm 0.04$ & $4.23 \pm 0.36$ & $6.86 \pm 0.31$ & $32.46 \pm 1.09$ & $12.02 \pm 0.45$ & $4.93 \pm 0.17$ & $4.10 \pm 0.05$ & $0.47 \pm 0.05$ & $76.58 \pm 5.43$ & $12.62 \pm 0.97$ & $4.08 \pm 0.51$ & $3.18 \pm 0.38$ & $85.68 \pm 1.56$ & $121.32 \pm 1.66$ \\
\hline 21 & $53.55 \pm 2.78$ & $45.18 \pm 4.15$ & $93.70 \pm 4.98$ & $5.76 \pm 0.22$ & $5.07 \pm 0.23$ & $0.69 \pm 0.04$ & $3.76 \pm 0.36$ & $6.12 \pm 0.31$ & $28.20 \pm 1.09$ & $10.91 \pm 0.45$ & $4.76 \pm 0.17$ & $4.04 \pm 0.05$ & $0.53 \pm 0.05$ & $76.96 \pm 5.43$ & $11.94 \pm 0.97$ & $5.50 \pm 0.51$ & $3.50 \pm 0.38$ & $84.03 \pm 1.56$ & $123.12 \pm 1.66$ \\
\hline 22 & $58.47 \pm 2.78$ & $51.68 \pm 4.15$ & $81.60 \pm 4.98$ & $7.41 \pm 0.22$ & $4.33 \pm 0.23$ & $0.66 \pm 0.04$ & $2.78 \pm 0.36$ & $4.84 \pm 0.31$ & $30.35 \pm 1.09$ & $10.32 \pm 0.45$ & $5.32 \pm 0.17$ & $4.16 \pm 0.05$ & $0.58 \pm 0.05$ & $90.86 \pm 5.43$ & $14.84 \pm 0.97$ & $5.88 \pm 0.51$ & $3.65 \pm 0.38$ & $84.38 \pm 1.56$ & $120.92 \pm 1.66$ \\
\hline 23 & $57.01 \pm 2.78$ & $47.43 \pm 4.15$ & $80.78 \pm 4.98$ & $5.36 \pm 0.22$ & $4.47 \pm 0.23$ & $0.60 \pm 0.04$ & $3.00 \pm 0.36$ & $5.07 \pm 0.31$ & $26.28 \pm 1.09$ & $9.61 \pm 0.45$ & $4.62 \pm 0.17$ & $4.12 \pm 0.05$ & $0.51 \pm 0.05$ & $77.14 \pm 5.43$ & $12.21 \pm 0.97$ & $5.21 \pm 0.51$ & $3.55 \pm 0.38$ & $82.63 \pm 1.56$ & $119.42 \pm 1.66$ \\
\hline 24 & $63.07 \pm 2.78$ & $66.04 \pm 4.15$ & $75.95 \pm 4.99$ & $4.83 \pm 0.23$ & $5.04 \pm 0.23$ & $0.60 \pm 0.04$ & $2.97 \pm 0.36$ & $5.44 \pm 0.31$ & $28.23 \pm 1.09$ & $10.04 \pm 0.45$ & $4.98 \pm 0.17$ & $3.98 \pm 0.05$ & $0.62 \pm 0.05$ & $64.91 \pm 5.44$ & $11.71 \pm 0.98$ & $5.05 \pm 0.51$ & $4.09 \pm 0.38$ & $83.79 \pm 1.56$ & $119.81 \pm 1.66$ \\
\hline 25 & $58.88 \pm 2.78$ & $62.99 \pm 4.15$ & $80.22 \pm 4.99$ & $5.80 \pm 0.23$ & $5.10 \pm 0.23$ & $0.62 \pm 0.04$ & $3.93 \pm 0.36$ & $6.01 \pm 0.31$ & $29.80 \pm 1.09$ & $11.02 \pm 0.45$ & $4.79 \pm 0.17$ & $4.09 \pm 0.05$ & $0.49 \pm 0.05$ & $68.48 \pm 5.44$ & $12.75 \pm 0.98$ & $4.02 \pm 0.51$ & $3.61 \pm 0.38$ & $82.29 \pm 1.56$ & $116.61 \pm 1.66$ \\
\hline
\end{tabular}


Tab. 3. Correlation matrix among characteristics studied

\begin{tabular}{|c|c|c|c|c|c|c|c|c|c|c|c|c|c|c|c|c|c|c|}
\hline & Yield & NF & FW & $\mathrm{FL}$ & FD & PT & NC & LletW & $\mathrm{LL}$ & LletL & TSS & $\mathrm{pH}$ & TA & PHeight & NNS & NT & NFlo & DFlow \\
\hline $\mathrm{NF}$ & 0.21 & & & & & & & & & & & & & & & & & \\
\hline FW & -0.06 & -0.87 & & & & & & & & & & & & & & & & \\
\hline FL & -0.08 & -0.75 & 0.72 & & & & & & & & & & & & & & & \\
\hline FD & -0.27 & -0.85 & 0.76 & 0.51 & & & & & & & & & & & & & & \\
\hline PT & -0.21 & -0.77 & 0.69 & 0.66 & 0.70 & & & & & & & & & & & & & \\
\hline $\mathrm{NC}$ & -0.34 & -0.49 & 0.52 & 0.20 & 0.62 & 0.18 & & & & & & & & & & & & \\
\hline LletW & -0.04 & 0.01 & 0.11 & 0.11 & 0.04 & -0.32 & 0.24 & & & & & & & & & & & \\
\hline LL & -0.03 & -0.14 & 0.20 & 0.21 & 0.21 & 0.01 & 0.20 & 0.38 & & & & & & & & & & \\
\hline LletL & -0.16 & -0.16 & 0.15 & 0.40 & 0.09 & -0.02 & 0.11 & 0.81 & 0.56 & & & & & & & & & \\
\hline TSS & 0.28 & 0.64 & -0.60 & -0.48 & -0.64 & -0.48 & -0.56 & -0.22 & -0.08 & -0.29 & & & & & & & & \\
\hline $\mathrm{pH}$ & -0.20 & -0.80 & 0.72 & 0.63 & 0.66 & 0.81 & 0.43 & -0.29 & 0.03 & -0.11 & -0.50 & & & & & & & \\
\hline $\mathrm{TA}$ & 0.14 & 0.77 & -0.79 & -0.70 & -0.66 & -0.61 & -0.41 & -0.25 & -0.38 & -0.43 & 0.63 & -0.65 & & & & & & \\
\hline PHeight & 0.13 & 0.10 & -0.06 & -0.27 & 0.04 & 0.03 & -0.09 & -0.41 & 0.31 & -0.44 & 0.42 & 0.09 & 0.11 & & & & & \\
\hline NNS & 0.18 & 0.05 & -0.02 & -0.26 & 0.11 & 0.02 & 0.00 & -0.44 & 0.29 & -0.49 & 0.37 & 0.09 & 0.16 & 0.97 & & & & \\
\hline NT & 0.13 & -0.11 & -0.06 & 0.13 & -0.04 & 0.21 & -0.26 & -0.44 & -0.17 & -0.31 & 0.15 & 0.18 & 0.14 & 0.26 & 0.24 & & & \\
\hline NFlo & 0.37 & 0.92 & -0.73 & -0.69 & -0.80 & -0.64 & -0.52 & -0.02 & -0.10 & -0.19 & 0.64 & -0.69 & 0.65 & 0.25 & 0.18 & -0.05 & & \\
\hline DFlow & -0.44 & -0.45 & 0.53 & 0.43 & 0.48 & 0.42 & 0.34 & 0.00 & 0.36 & 0.13 & -0.42 & 0.40 & -0.45 & 0.03 & 0.03 & -0.04 & -0.45 & \\
\hline DFP & -0.30 & -0.74 & 0.76 & 0.73 & 0.64 & 0.72 & 0.35 & 0.10 & 0.22 & 0.28 & -0.74 & 0.64 & -0.70 & -0.27 & -0.29 & 0.10 & -0.66 & 0.73 \\
\hline
\end{tabular}

Correlation coefficient significant at $\mathrm{P}=0.05$ with value $\geq 0.40$. TA: titratable acidity ( $\%$ of citric acid); TSS: total soluble solids (Brix); NF: number of fruit per plant; FW: mean of fruit weight per plant; FL: fruit length; FD: fruit diameter; PT: pericarp thickness; NC: number of carpel; LletW: leaflet width; LletL: leaflet length; LL: principal leaf length; PHeight: plant height; NNS: number of node on stem; NT: number of tiller; NFlo: number of floret; DFlow: number of days to flowering; DFP: number of days to fruit production

were mainly attributed towards climatic data during two years. Genotype $\times$ year interaction was only significant for some of the characters including number of fruit per plant, titratable acidity and for fruit yield which revealed that the evaluation experiments under field condition should be conducted over the years or locations to minimize errors (Gonçalves et al., 2003). The experimental coefficient of variation $(\mathrm{CV})$ varied from 2.74 to 18.55 . In general, $\mathrm{CV}$ value lower than $20 \%$ is considered to be good, indicating the accuracy of conducted experiments. Mean data revealed high range for most of studied traits (Tab. 2). Fruit yield ranged from 50.33 to $63.50 \mathrm{t} \mathrm{ha}^{-1}$, number of fruit per plant from 40.99 to 308.98 , mean of fruit weight per plant from 14.60 to 112.48 ; fruit length from 2.68 to 7.41 , fruit diameter from 2.82 to 5.56 , pericarp thickness from 0.36 to 0.83 , nb from 2.30 to 4.95 , leaflet width from 4.72 to 6.86 , principal leaf length from 24.71 to 32.46 , leaflet length from 8.25 to 12.02 , total soluble solids from 4.34 to $5.32, \mathrm{pH}$ from 4.04 to 4.32 , titratable acidity from 0.45 to 0.85 , plant height from 64.91 to 168.03 , number of node on stem from 10.76 to 26.43 , number of tillers from 4.02 to 5.88 , number of floret from 2.66 to 7.92 , number of days to flowering from 81.33 to 87.49 and number of days to fruit production from 107.67 to 127.97 (Tab. 2). Wide range of phenotypic variability for all the traits could be exploited for initiating of breeding programs to develop new high yielding tomato genotypes. Maximum and minimum variability were observed for number of fruit per plant and $\mathrm{pH}$ respectively. Different genotypes present the high value for studied traits. Genotype 15 showed the low value for mean of fruit weight per plant, fruit length, fruit diameter, pericarp thickness, number of days to flowering and number of days to fruit production and high value for titratable acidity, number of floret and number of fruit per plant.

In the present study, negative association between fruit yield and number of days to flowering was observed. Based on this association the genotype with short vegetative growth period produces high yield (Tab. 3).

Total soluble solids has a negative correlation with mean of fruit weight per plant, fruit length, fruit diameter, pericarp thickness and number of carpel and positive correlation with number of fruit per plant. Total soluble solids did not show any correlation with fruit yield. Hierarchical cluster analysis allowed the assessment of similarity and clarified some of the relationships among tomato genotypes. UPGMA produced a dendrogram with four clusters (Fig. 1). The first cluster included $64 \%$ of studied tomato genotypes. The second cluster was consisted of the genotypes 4, 5, 7, 19, 20 and 25. The third and fourth clusters each were composed of one genotype. The reason of such a low variability can be explained with the fact that tomato is mostly self-pollinated. Principal component analysis was used to identify the most significant variables in the data set. Previously, PCA had been used to evaluate germplasm of different species: olive (Cantini et al., 1999), pomegranate (Mars and Marrakchi, 1999), loquat (Badenes et al., 2000; Martinez-Calvo et al., 2008) and apricot (Ruiz and Egea, 2008). It was also used for establishing genetic relationships among cultivars and to study correlations 
Tab. 4. Eigen values and proportion of variance explained by the 7 principal components with respect to 25 tomato germplasm traits

\begin{tabular}{|c|c|c|c|c|c|c|c|c|c|c|c|c|c|c|}
\hline & \multicolumn{14}{|c|}{ PC axis } \\
\hline & 1 & & 2 & & 3 & & 4 & & 5 & & 6 & & 7 & \\
\hline Eigen-values & 8.16 & & 3.29 & & 2.00 & & 1.41 & & 1.11 & & 0.68 & & 0.60 & \\
\hline $\begin{array}{l}\text { Explained proportion } \\
\text { of variation (\%) }\end{array}$ & 0.43 & & 0.17 & & 0.11 & & 0.07 & & 0.06 & & 0.04 & & 0.03 & \\
\hline $\begin{array}{c}\text { Cumulative proportion } \\
\text { of variation (\%) }\end{array}$ & 43 & & 60 & & 71 & & 78 & & 84 & & 88 & & 91 & \\
\hline \multirow[t]{2}{*}{ Character } & \multicolumn{14}{|c|}{ Eigen vectors } \\
\hline & Value & $\mathrm{r}$ & Value & $\mathrm{r}$ & Value & $\mathrm{r}$ & Value & $\mathrm{r}$ & Value & $\mathrm{r}$ & Value & $\mathrm{r}$ & Value & $\mathrm{r}$ \\
\hline Yield & 0.12 & 0.33 & 0.07 & 0.13 & -0.01 & -0.02 & 0.51 & 0.61 & 0.55 & 0.58 & -0.29 & -0.24 & 0.37 & 0.29 \\
\hline NF & 0.33 & $0.93^{\circ}$ & -0.10 & -0.17 & -0.05 & -0.07 & -0.06 & -0.07 & -0.14 & -0.15 & -0.18 & -0.15 & 0.08 & 0.06 \\
\hline FW & -0.31 & -0.89 & 0.06 & 0.11 & -0.05 & -0.07 & 0.08 & 0.10 & 0.22 & 0.23 & -0.21 & -0.17 & 0.07 & 0.05 \\
\hline FL & -0.28 & -0.79 & -0.03 & -0.05 & 0.08 & 0.11 & 0.37 & 0.44 & -0.07 & -0.08 & 0.03 & 0.02 & -0.12 & -0.09 \\
\hline FD & -0.30 & -0.85 & 0.11 & 0.19 & -0.09 & -0.13 & -0.16 & -0.19 & 0.18 & 0.19 & 0.15 & 0.12 & 0.00 & 0.00 \\
\hline PT & -0.27 & -0.77 & 0.22 & 0.39 & 0.14 & 0.20 & 0.12 & 0.15 & -0.09 & -0.09 & -0.11 & -0.09 & -0.30 & -0.23 \\
\hline $\mathrm{NC}$ & -0.20 & -0.57 & -0.05 & -0.10 & -0.17 & -0.23 & -0.47 & -0.56 & 0.29 & 0.31 & 0.21 & 0.17 & 0.31 & 0.24 \\
\hline LletW & -0.04 & -0.12 & -0.46 & -0.84 & -0.24 & -0.34 & 0.08 & 0.09 & 0.13 & 0.14 & 0.18 & 0.15 & 0.13 & 0.10 \\
\hline $\mathrm{LL}$ & -0.09 & -0.25 & -0.09 & -0.16 & -0.60 & -0.85 & 0.20 & 0.23 & -0.17 & -0.18 & 0.06 & 0.05 & 0.03 & 0.02 \\
\hline LletL & -0.11 & -0.31 & -0.43 & -0.78 & -0.21 & -0.30 & 0.27 & 0.32 & -0.13 & -0.14 & 0.24 & 0.20 & -0.17 & -0.13 \\
\hline TSS & 0.27 & 0.77 & 0.13 & 0.24 & -0.11 & -0.15 & 0.16 & 0.19 & -0.09 & -0.10 & 0.07 & 0.06 & -0.35 & -0.27 \\
\hline $\mathrm{pH}$ & -0.28 & -0.79 & 0.23 & 0.42 & 0.09 & 0.12 & 0.01 & 0.01 & 0.09 & 0.09 & -0.02 & -0.01 & -0.18 & -0.14 \\
\hline TA & 0.30 & 0.85 & 0.06 & 0.10 & 0.13 & 0.18 & -0.16 & -0.20 & -0.12 & -0.12 & 0.13 & 0.11 & 0.18 & 0.14 \\
\hline PHeight & 0.07 & 0.20 & 0.41 & 0.74 & -0.43 & -0.60 & 0.02 & 0.02 & -0.02 & -0.02 & 0.03 & 0.03 & -0.07 & -0.06 \\
\hline NNS & 0.06 & 0.18 & 0.42 & 0.77 & -0.41 & -0.58 & -0.03 & -0.04 & 0.08 & 0.09 & 0.06 & 0.05 & 0.01 & 0.01 \\
\hline NT & 0.01 & 0.03 & 0.30 & 0.55 & 0.20 & 0.28 & 0.34 & 0.40 & -0.27 & -0.28 & 0.58 & 0.48 & 0.48 & 0.37 \\
\hline NFlo & 0.31 & 0.87 & -0.03 & -0.05 & -0.10 & -0.14 & 0.09 & 0.10 & -0.05 & -0.05 & -0.32 & -0.26 & 0.12 & 0.09 \\
\hline DFlow & -0.22 & -0.63 & 0.04 & 0.07 & -0.17 & -0.24 & -0.15 & -0.18 & -0.51 & -0.53 & -0.40 & -0.33 & 0.30 & 0.23 \\
\hline DFP & -0.31 & -0.88 & -0.02 & -0.04 & 0.07 & 0.10 & 0.09 & 0.11 & -0.26 & -0.28 & -0.22 & -0.18 & 0.30 & 0.23 \\
\hline
\end{tabular}

Correlation coefficient significant at $P=0.05$ with value $\geq 0.0 .39$. TA: titratable acidity (\% of citric acid); TSS: total soluble solids (Brix); NF: number of fruit per plant; FW: mean of fruit weight per plant; FL: fruit length; FD: fruit diameter; PT: pericarp thickness; NC: number of carpel; LletW: leaflet width; LletL: leaflet length; LL: principal leaf length; PHeight: plant height; NNS: number of node on stem; NT: number of tiller; NFlo: number of floret; DFlow: number of days to flowering; DFP: number of days to fruit production

among fruit traits within sets of peach genotypes (Esti et al., 1997; Wu et al., 2003) and apricot genotypes (Badenes et al., 1998; Gurrieri et al., 2001; Azodanlou et al., 2003). Associations between traits emphasized by this method may correspond to genetic linkage between loci controlling traits or a pleiotropic effect (Iezzoni and Pritts, 1991). Results from the PCA indicated that more than $91 \%$ of the variability observed can be explained by the first seven components (Tab. 4). Correlation between the original variables and the seven principal components were shown in Tab. 4. Variables with higher scores on PC1 are: mean of fruit weight per plant, fruit length, fruit diameter, pericarp thickness, number of carpel, $\mathrm{pH}$, number of days to flowering and number of days to fruit production. PC2 includes $\mathrm{pH}$, plant height, number of node on stem and number of tillers. The variables that show high correlation with $\mathrm{PC} 1$ can be considered as representatives of fruit size, while properties that show high correlation with PC2 are the ones related to plant characteristics. This type of analy- sis essentially restructured the data sets containing many correlated variables into smaller sets of components.

\section{Conclusions}

The study revealed considerable phenotypical (and presumably genetic) diversity among tomato genotypes. The cluster analysis grouped the genotypes according to four groups. A high correlation was found between some traits and principal components, which could reduce the number of traits to be studied in tomato germplasm.

\section{References}

Agong SG, Schittenhelm S, Friedt W (2000). Genotypic variation of Kenyan tomato (Lycopersicon esculentum L.) germplasm. Plant Genetic Resources Newsletter 123:61-67.

Azodanlou R, Darbellay C, Luisier JL, Villettaz JC, Amado R (2003). Development of a model for quality assessment of tomatoes and apricots. Food Sci Technol 36:223-233. 
158

Badenes ML, Martınez-Calvo J, Llacer G (2000). Analysis of a germplasm collection of loquat (Eriobotrya japonica Lindl.). Euphytica 114:187-194.

Badenes ML, Martınez-Calvo J, Llacer G (1998). Analysis of an apricot germplasm from the European ecogeographical group. Euphytica 102:93-99.

Cantini C, Cimato A, Sani G (1999). Morphological evaluation of olive germplasm present in Tuscany region. Euphytica 109:173-181.

Carelli BP, Gerald LTS, Grazziotin FG, Echeverrigaray S (2006). Genetic diversity among Brazilian cultivars and landraces of tomato Lycopersicon esculentum Mill. revealed by RAPD markers. Genetic Resources and Crop Evolution 53:395-400.

Esti M, Messia MC, Sinesio F, Nicotra A, Conte L (1997). Quality evaluation of peaches and nectarines by electrochemical and multivariate analyses: relationships between analytical measurements and sensory attributes. Food Chem 60:659-666.

Gonçalves PDS, Bortoletto N, Martins ALM, Costa RBD, Gallo PB (2003). Genotype-environment interaction and phenotypic stability for girth growth and rubber yield of Hevea clones in São Paulo State, Brazil. Genetics and Molecular Biology 26:441-448.

Gurrieri F, Audergon JM, Albagnac G, Reich M (2001). Soluble sugars and carboxylic acids in ripe apricot fruit as parameters for distinguishing different cultivars. Euphytica 117:183189.

Iezzoni AF, Pritts MP (1991). Applications of principal components analysis to horticultural research. HortSci 26:334-338.

Mars M, Marrakchi M (1999). Diversity of pomegranate (Punica granatum L.) germplasm in Tunisia. Genet Resour Crop Evol 46:461-467.
Michaelson MJ, Price HJ, Ellison JR, Johnston JS (1991). Comparison of plant DNA contents determined by feulgen microspectrophotometry and laser flow cytometry. American Journal of Botany 78:183-188.

Miller JC, Tanksley SD (1990). RFLP analysis of phylogenetic relationships and genetic variation in the genus Lycopersicon. Theor Appl Genet 80:437-448.

Rick CM (1991). Tomato paste: a concentrated review of genetic highlights from the beginning to the advent of molecular genetics. Genetics 128:1-5.

Rick CM (1976). Tomato, p. 269-273. In: Simmonds NW (Ed.). Evolution of Crop Plants. Longman, London.

Ruiz D, Egea J (2008). Phenotypic diversity and relationships of fruit quality traits in apricot (Prunus armeniaca L.) germplasm. Euphytica 163: 143-158.

Singh JK, Singh JP, Jain SK, Aradhana J, Joshi A (2002). Studies on genetic variability and its importance in tomato (Lycopersicon esculentum Mill.). Prog Hort 34:77-79.

Van der Hoeven R, Ronning C, Giovannoni J, Martin G, Tanksley SD (2002). Deductions about the number, organization, and evolution of genes in the tomato genome based on analysis of a large expressed sequence tag collection and selective genomic sequencing. The Plant Cell 14:14411456.

Williams CE, St. Clair DA (1993). Phenetic relationships and levels of variability detected by restriction fragment length polymorphism and random amplified polymorphic DNA analysis of cultivated and wild accessions of Lycopersicon esculentum. Genome 36:619-630.

Wu B, Quilot B, Kervella J, Genard M, Li S (2003). Analysis of genotypic variation of sugar and acid contents in peaches and nectarines through the principle component analysis. Euphytica 132:375-384. 